\title{
Solid acid catalytic conversion of oil shale: Effects of sulfonic acid grafting on oil yield enhancing and quality improvement
}

\author{
Xianglong Meng ${ }^{a, b}$, Zhilei Qi ${ }^{c}$, Cong Yuc ${ }^{c}$,Junjie Bian*c, Zhongliang Ma ${ }^{a, b}$, Qiulian Long ${ }^{a, b}$, Jianzheng Su ${ }^{a, b}$
}

a State Research and Development Center for Oil Shale Exploitation, SINOPEC, Beijing, China.

b Sinopec Petroleum Exploration and Production Research Institute, SINOPEC, Beijing, China.

c Key Laboratory of Marine Chemistry Theory and Technology of Ministry of Education, Ocean University of China, Qingdao, China.

\author{
Corresponding Author Junjie Bian*_Email: junjiebian@ouc.edu.cn \\ Address: Key Laboratory of Marine Chemistry Theory and Technology of Ministry of Education, Ocean University of China, Qingdao, China.
}




\subsection{Catalyst preparation}

Clin/SBA-15 $1 \mathrm{~g}$ and (3-mercaptopropyl)trimethoxysilane (MPTMS) $0.79 \mathrm{~g}$ were dissolved in toluene $50 \mathrm{~mL}$, in a three-necked flask. Then, it was placed into an oil bath with a magnetic stirrer and a thermocouple. The reaction of these monomers was carried out at $110{ }^{\circ} \mathrm{C}$ for $24 \mathrm{~h}$ under refluxing and continuous stirring. The obtained product, SH/SBA-15 was washed with ethanol for several times to remove MPTMS. The solid was immersed in $\mathrm{H} 2 \mathrm{O} 2 \mathrm{under}$ stirring for $12 \mathrm{~h}$ at room temperature. Then the filtered solid was dissolved in $\mathrm{H} 2 \mathrm{SO} 40.5 \mathrm{~mol} / \mathrm{L} 50 \mathrm{~mL}$ under stirring for $12 \mathrm{~h}$ at room temperature. The solid products were washed and filtered with ethanol and water. At the same time, the catalyst sulfonic acid groups $4 \mathrm{mmol} / \mathrm{g} \mathrm{SO} \mathrm{H} / \mathrm{SBA}-15[\mathrm{SO} 3 \mathrm{H} / \mathrm{SBA}-15(4)]$ was synthesized in the same procedures as above. 
Table S1 Geochemical properties of oil shale

(determined by golden tube testing and Rock-Eval pyrolysis)

\begin{tabular}{|c|c|c|c|c|c|c|c|c|}
\hline \multirow{2}{*}{$\begin{array}{c}T \max \\
\left({ }^{\circ} \mathrm{C}\right)\end{array}$} & TOC & Moisture & $\mathrm{PC}$ & $\mathrm{RC}$ & S1 & $\mathrm{S} 2$ & $I_{\mathrm{H}}$ & $I_{\mathrm{O}}$ \\
\hline & & $(\%)$ & & & $\left(\mathrm{mg} / \mathrm{g}_{\mathrm{or}}\right.$ & $\left(\mathrm{mg} / \mathrm{g}_{\text {ore }}\right)$ & ) & ) \\
\hline 433 & 17.89 & $\begin{array}{r}13.53 \\
0\end{array}$ & 11.0 & 6.89 & 1.54 & 127.37 & 712 & 21 \\
\hline
\end{tabular}


Table S2 Characteristics of oil shale determine by Fisher assay analysis under anoxic conditions

\begin{tabular}{ll}
\hline Fisher Assay & Weight (\%) \\
\hline Oil & 11.82 \\
Water & 2.86 \\
Residue & 75.11 \\
Gas+Loss & 10.21 \\
\hline
\end{tabular}


Table S3 Products yield of oil shale conversion with $\mathrm{SO}_{3} \mathrm{H} / \mathrm{SBA}-15$

\begin{tabular}{ccc}
\hline & Oil $(\mathrm{g})$ & Residue $(\mathrm{g})$ \\
\hline $1 \mathrm{mmol} / \mathrm{g}$ & 0.4754 & 6.9657 \\
$2 \mathrm{mmol} / \mathrm{g}$ & 0.5130 & 6.9560 \\
$3 \mathrm{mmol} / \mathrm{g}$ & 0.5810 & 6.9514 \\
$4 \mathrm{mmol} / \mathrm{g}$ & 0.6666 & 6.9453 \\
$5 \mathrm{mmol} / \mathrm{g}$ & 0.6646 & 6.9487 \\
$6 \mathrm{mmol} / \mathrm{g}$ & 0.6592 & 6.9495 \\
\hline
\end{tabular}


Table S4 Components of $\mathrm{SO}_{3} \mathrm{H} / \mathrm{SBA}-15$ catalyst for catalytic conversion reaction of oil shale

\begin{tabular}{|c|c|c|c|c|c|c|c|c|c|}
\hline \multirow{2}{*}{$\begin{array}{l}\text { Retention time } \\
\qquad(\min )\end{array}$} & \multirow{2}{*}{ Compound } & \multirow{2}{*}{ Molecular Formula } & \multirow{2}{*}{$\begin{array}{l}\text { Boiling point } \\
\qquad\left({ }^{\circ} \mathrm{C}\right)\end{array}$} & \multirow[b]{2}{*}{$1 \mathrm{mmol} / \mathrm{g}$} & \multicolumn{3}{|c|}{ Weight (\%) } & \multirow[b]{2}{*}{$5 \mathrm{mmol} / \mathrm{g}$} & \multirow[b]{2}{*}{$6 \mathrm{mmol} / \mathrm{g}$} \\
\hline & & & & & $2 \mathrm{mmol} / \mathrm{g}$ & $3 \mathrm{mmol} / \mathrm{g}$ & $4 \mathrm{mmol} / \mathrm{g}$ & & \\
\hline 4.421 & Phenol & $\mathrm{C}_{6} \mathrm{H}_{6} \mathrm{O}$ & 182 & 2.01 & 1.96 & - & 2.28 & 2.07 & 1.49 \\
\hline 4.910 & $\begin{array}{l}\text { 2-Cyclpenten-1-one,2,3- } \\
\text { dimethil- }\end{array}$ & $\mathrm{C}_{7} \mathrm{H}_{10} \mathrm{O}$ & - & 4.78 & 4.02 & 7.84 & 10.38 & 10.27 & 10.00 \\
\hline 4.914 & 2,4-Hexadiene,2,5-dimethyl- & $\mathrm{C}_{8} \mathrm{H}_{14}$ & 135 & 5.81 & 4.46 & 4.32 & 7.90 & 5.20 & 5.13 \\
\hline 5.077 & Naphthalene,decahydro-,cis- & $\mathrm{C}_{10} \mathrm{H}_{18}$ & 185 & 7.92 & 6.26 & 12.10 & 8.40 & 3.89 & 4.66 \\
\hline 5.376 & Naphthalene,decahydro-,cis- & $\mathrm{C}_{10} \mathrm{H}_{18}$ & 185 & 1.09 & - & 2.94 & 1.39 & 3.44 & 4.94 \\
\hline 5.575 & Phenol,2,5-dimethyl- & $\mathrm{C}_{8} \mathrm{H}_{10} \mathrm{O}$ & 211.5 & 3.38 & 2.97 & 1.94 & 1.70 & 1.03 & 1.74 \\
\hline 5.606 & Benzoic acid & $\mathrm{C}_{7} \mathrm{H}_{6} \mathrm{O}_{2}$ & 249 & 4.58 & 9.05 & - & 10.91 & 3.01 & 5.15 \\
\hline 5.682 & $\begin{array}{c}\text { 1,6-Dimethylhepta-1,3,5- } \\
\text { triene }\end{array}$ & $\mathrm{C}_{9} \mathrm{H}_{14}$ & 164.8 & 6.89 & 11.62 & 12.70 & 25.02 & 16.46 & 8.22 \\
\hline 5.877 & $\begin{array}{c}\text { 2-Octen-1-ol,3,7- } \\
\text { dimethyl-,isobutyrate,(Z)- }\end{array}$ & $\mathrm{C}_{14} \mathrm{H}_{26} \mathrm{O}_{2}$ & 282.2 & 4.90 & 4.84 & 1.62 & 0.74 & 0.85 & 0.98 \\
\hline 5.964 & $\begin{array}{c}\text { 3,7-Dimethyl-2,6-nonadien-1- } \\
\text { ol }\end{array}$ & $\mathrm{C}_{11} \mathrm{H}_{20} \mathrm{O}$ & 244.9 & 3.05 & 4.83 & 5.77 & 5.64 & 2.35 & 3.48 \\
\hline
\end{tabular}




\begin{tabular}{|c|c|c|c|c|c|c|c|c|c|}
\hline 6.360 & Undecane,2-methyl- & $\mathrm{C}_{12} \mathrm{H}_{26}$ & 170 & 7.87 & 5.91 & 5.62 & 3.56 & 2.01 & 3.44 \\
\hline 6.398 & 1-Dodecanol,3,7,11-trimethyl- & $\mathrm{C}_{15} \mathrm{H}_{32} \mathrm{O}$ & 279.2 & 3.64 & 2.75 & 2.07 & 0.37 & 4.24 & 3.29 \\
\hline 6.456 & Tetradecane,2,6,10-trimethyl- & $\mathrm{C}_{17} \mathrm{H}_{36}$ & 282 & 1.94 & - & - & 2.61 & 2.41 & - \\
\hline 6.619 & Tetradecane,2,6,10-trimethyl- & $\mathrm{C}_{17} \mathrm{H}_{36}$ & 282 & 1.32 & 1.59 & - & 0.48 & 0.28 & 1.01 \\
\hline 7.006 & Tetradecane,2,6,10-trimethyl- & $\mathrm{C}_{17} \mathrm{H}_{36}$ & 282 & 0.88 & - & 5.11 & - & 1.95 & 0.37 \\
\hline 7.011 & Dodecane,5,8-diethyl- & $\mathrm{C}_{16} \mathrm{H}_{34}$ & - & 5.02 & 1.24 & 4.80 & - & 5.92 & 3.92 \\
\hline 7.354 & Tetradecane,2,6,10-trimethyl- & $\mathrm{C}_{17} \mathrm{H}_{36}$ & 282 & 0.89 & 0.17 & 2.70 & 1.73 & 2.19 & - \\
\hline 7.504 & $\begin{array}{c}\text { 7-Methyl-Z-tetradecen-1- } \\
\text { olacetate }\end{array}$ & $\mathrm{C}_{17} \mathrm{H}_{32} \mathrm{O}_{2}$ & - & 1.65 & 1.28 & 2.63 & - & 2.04 & 1.93 \\
\hline 7.565 & Tetradecane,2,6,10-trimethyl- & $\mathrm{C}_{17} \mathrm{H}_{36}$ & 282 & - & 2.40 & - & 1.38 & 1.90 & 4.95 \\
\hline 7.809 & 2-Hexadecanol & $\mathrm{C}_{16} \mathrm{H}_{34} \mathrm{O}$ & 135 & 1.21 & 0.73 & 0.25 & 0.66 & 3.03 & 4.05 \\
\hline 8.045 & Hexadecane & $\mathrm{C}_{16} \mathrm{H}_{34}$ & 288.7 & 0.63 & 0.46 & 0.31 & 0.58 & 1.00 & 2.03 \\
\hline 8.172 & Tetradecane,2,6,10-trimethyl- & $\mathrm{C}_{17} \mathrm{H}_{36}$ & 282 & 2.84 & 2.95 & - & - & 1.99 & 1.82 \\
\hline 8.878 & Tetradecane,2,6,10-trimethyl- & $\mathrm{C}_{17} \mathrm{H}_{36}$ & 282 & 3.69 & - & 1.69 & - & - & - \\
\hline 9.009 & Tetradecane,2,6,10-trimethyl- & $\mathrm{C}_{17} \mathrm{H}_{36}$ & 282 & - & 3.05 & - & 1.30 & - & - \\
\hline 9.226 & 1-Dodecanol,3,7,11-trimethyl- & $\mathrm{C}_{15} \mathrm{H}_{32} \mathrm{O}$ & 279.2 & 2.12 & 2.32 & 2.07 & 1.90 & 1.94 & 2.30 \\
\hline 10.753 & Tetradecane,2,6,10-trimethyl- & $\mathrm{C}_{17} \mathrm{H}_{36}$ & 282 & - & 2.22 & - & 0.94 & - & 0.29 \\
\hline
\end{tabular}




\begin{tabular}{|c|c|c|c|c|c|c|c|c|c|}
\hline 11.437 & Tetradecanoic acid & $\mathrm{C}_{14} \mathrm{H}_{28} \mathrm{O}_{2}$ & 250 & 3.77 & 3.96 & 4.82 & 2.65 & 0.90 & - \\
\hline 11.447 & Tridecanoic acid & $\mathrm{C}_{13} \mathrm{H}_{26} \mathrm{O}_{2}$ & 236 & 2.58 & 6.07 & 5.46 & 2.65 & 2.39 & 5.56 \\
\hline 11.459 & Tetradecanoic acid & $\mathrm{C}_{14} \mathrm{H}_{28} \mathrm{O}_{2}$ & 250 & 0.99 & 1.02 & 1.38 & - & 2.28 & 3.81 \\
\hline 11.908 & Dodecanoic acid,ethylester & $\mathrm{C}_{14} \mathrm{H}_{28} \mathrm{O}_{2}$ & 269 & 1.32 & 0.97 & 1.44 & - & 6.53 & 7.58 \\
\hline 13.430 & Dodecane,2,6,10-trimethyl- & $\mathrm{C}_{15} \mathrm{H}_{32}$ & 249.1 & 2.36 & 1.52 & 2.42 & 0.89 & - & 2.67 \\
\hline 15.088 & Tetradecane,2,6,10-trimethyl- & $\mathrm{C}_{17} \mathrm{H}_{36}$ & 282 & 1.06 & - & - & - & 1.19 & - \\
\hline 15.094 & Heptadecane & $\mathrm{C}_{17} \mathrm{H}_{36}$ & 302 & 1.37 & 0.82 & 0.49 & 0.97 & 1.02 & - \\
\hline 16.914 & Nonadecane & $\mathrm{C}_{19} \mathrm{H}_{40}$ & 330 & 2.30 & 2.01 & 1.62 & 0.53 & 0.88 & 0.92 \\
\hline 18.888 & Tetradecane,2,6,10-trimethyl- & $\mathrm{C}_{17} \mathrm{H}_{36}$ & 282 & - & 0.99 & - & - & 1.23 & - \\
\hline 18.898 & Nonadecane & $\mathrm{C}_{19} \mathrm{H}_{40}$ & 330 & - & 1.90 & 0.69 & - & - & 0.99 \\
\hline 21.000 & Nonadecane & $\mathrm{C}_{19} \mathrm{H}_{40}$ & 330 & 3.31 & - & 2.38 & - & 1.29 & - \\
\hline 23.112 & Tetradecane,2,6,10-trimethyl- & $\mathrm{C}_{17} \mathrm{H}_{36}$ & 282 & - & 0.68 & 0.34 & - & - & - \\
\hline 25.201 & Dodecane,5,8-diethyl- & $\mathrm{C}_{16} \mathrm{H}_{34}$ & - & - & - & 1.31 & - & 0.93 & 1.21 \\
\hline 25.224 & Nonadecane & $\mathrm{C}_{19} \mathrm{H}_{40}$ & 330 & 1.94 & 2.05 & 1.66 & 0.80 & 1.28 & 1.34 \\
\hline 25.555 & $\begin{array}{c}\text { E-8-Methyl-9-tetradecen-1- } \\
\text { olacetate }\end{array}$ & $\mathrm{C}_{17} \mathrm{H}_{32} \mathrm{O}_{2}$ & 328.5 & 0.89 & 0.93 & 0.73 & 0.42 & 0.61 & 0.73 \\
\hline
\end{tabular}

